\title{
Transverse Injection into Subsonic Crossflow with Various Injector Orifice Geometries
}

\author{
Lancert E. Foster ${ }^{1}$ and Khairul B. Zaman. ${ }^{2}$ \\ NASA Glenn Research
}

Computational and experimental results are presented for a case study of single injectors employed in $90^{\circ}$ transverse injection into a non-reacting subsonic flow. Different injector orifice shapes are used (including circular, square, diamond-shaped, and wide rectangular slot), all with constant cross-sectional area, to observe the effects of this variation on injector penetration and mixing. Whereas the circle, square, and diamond injector produce similar jet plumes, the wide rectangular slot produces a plume with less vertical penetration than the others. There is also some evidence that the diamond injector produces slightly faster penetration with less mixing of the injected fluid. In addition, a variety of rectangular injectors were analyzed, with varying length/width ratios. Both experimental and computational data show improved plume penetration with increased streamwise orifice length. 3-D Reynolds-Averaged Navier-Stokes (RANS) results are obtained for the various injector geometries using NCC (National Combustion Code) with the $\kappa-\varepsilon$ turbulence model in multi-species modes on an unstructured grid. Grid sensitivity results are also presented which indicate consistent qualitative trends in the injector performance comparisons with increasing grid refinement.

\section{Introduction}

Tluidic injection into crossflow has a wide variety of applications, such a film cooling, injection/combustion, 1 boundary layer control, and thrust vectoring. The configuration of the injector is dictated by the desired application. Thus, it is important to obtain an understanding of how the performance characteristics (i.e., penetration, mixing, and induced wall force) are affected by injector configuration parameters, such as: orifice geometry, orientation relative to primary flow, injector-to-freestream pressure ratios, etc. This study is concerned with the effects of varying orifice geometry in injectors oriented at $90^{\circ}$ to the cross flow.

The present study is focused on understanding the effects of variations of orifice geometry on the performance of flush-mounted injectors for a subsonic flow. The primary performance criterion was penetration. The injector-tofreestream velocity ratio is roughly 2.5:1. Experimental results of the subsonic jet-in-crossflow are presented and supported with a matching cfd results.

\section{Methodology}

\section{A. Experiment}

\footnotetext{
${ }^{1}$ Aerospace Engineer, Inlet and Nozzle Branch, 21000 Brookpark Rd MS: 5-12.

${ }^{2}$ Aerospace Engineer, Inlet and Nozzle Branch, 21000 Brookpark Rd MS 5-12..
} 
Testing was performed in NASA Glenn's CW-15 Low Speed Wind Tunnel with a single injector in the duct floor, oriented at $90^{\circ}$ to the crossflow [Fig. 1]. A single glass plate (0.5 in thick) contained all the injector orifices used, with all holes being covered except for the current injector being tested [Fig. 2]. Injector orifice shapes included circle, square, diamond, and rectangles of varying length/width ratios. All injectors had a cross-sectional area of $0.44 \mathrm{in}^{2}$ (the area of a circle 0.75 inches in diameter). Below the injector plate, there was a cylindrical plenum attached to a hose feeding pressurized air. The tunnel was operated with a cross flow of approximately 5 $\mathrm{m} / \mathrm{s}$, with air blowing through the injectors at approximately $12 \mathrm{~m} /$. Air velocity through the injectors was measured with a 1-D hotwire placed directly over the injector center at a height of 0 in. This same hotwire was used downstream of the injector to measure axial velocity at $\mathrm{x} / \mathrm{D}=5$ and $\mathrm{x} / \mathrm{D}=10$ (3.75 in and 7.5 in, respectively) where $\mathrm{D}$ is the 0.75 in, the diameter of the circular injector. At each downstream position, the hotwire was translated throughout that 2-D plane to record a full axial velocity field.

\section{B. CFD}

Unstructured grids were generated, matching the geometry of the experiment. Each grid consisted of three distinct regions [Fig 3]: one for the injector, one for the area around the injector plume, and one for the rest of the duct outside of the injector plume. The grid spacing was tighter in the regions for the injector and its jet plume, as these are the places where the largest gradients and crucial mixing behavior occur. Also, keeping the grid coarser in the region outside of the jet plume allows us to keep the grid less expensive by not wasting points in a region with rather calm behavior. The same grid scheme was used for all cases, with grid spacing kept constant, so as to make a fair comparison of the computational results. Each grid consisted of approximately 150,000 points at the finest level.

For this study, NCC (the National Combustion Code) is used. It is a 3-D RANS solver, employed primarily by the combustion branch at NASA Glenn, that allows for multiple block parallel processing. For this study, the $\kappa-\varepsilon$ turbulence model is used and the code is run in multi-species mode (with one species for the freestream and one for the injectant). While both species have the physical properties of air, multi-species running allows us to 'dye' the injected fluid. This way, one can plot mass fractions of injected fluid in the field, thus aiding the observation of injector performance.

\section{RESULTS}

\section{Primary Shapes}

Both experimental and computational data show similar penetration for the circle, diamond, and square injectors [Fig 4] at axial distances of $\mathrm{x} / \mathrm{D}=10$. The wide slot, on the other hand, clearly generates a plume with less penetration than the other three injectors. However, at $x / D=5$, [Fig 5], the diamond injector is showing slightly greater penetration than the circle and square, although the circle and square seem to catch up further downstream.

\section{Rectangular Slot Aspect Ratio Study}

All cases run consisted of injectors with rectangular orifice shapes with varying aspect ratios (axial length/width). Both experimental and cfd results show improve plume penetration with increased aspect ratio [Fig $6,7,8]$

The improved penetration is apparently largely due to a sustained generation of streamwise vorticity over a relatively long streamwise length. And this improved vorticity production is likely attributed to a "buffering effect." Specifically, the injector flow closer to the trailing edge of a longitudinal rectangular injector is better buffered from the axial momentum of the freestream by the streamwise vortices developing along the forward portion of the injector. In addition, injected flow closer to the leading edge is given some upward lift from the trailing edge injector flow blowing up from underneath.

As injector aspect ratio increases, the ratio of viscous surface area to fluid volume increases (cross-sectional area being held constant). This also causes the boundary layer within the injector to encompass an increasingly large proportion of the injector orifice area. Eventually, the viscous effects of increasing this surface area ratio would become more detrimental than the benefits of increasing the aspect ratio.

\section{Grid Convergence Study}


For all the cases in this study, grid independent results were not obtained. Consequently, the data acquired is primarily of qualitative value. As described earlier, coarse and fine grid solutions were obtained for each case. Whereas quantities could not be accurately computed, comparisons were made based on both grid levels to ensure the constancy of the observed trends. The trends observed in this study are all consistent at all grid resolutions. For future study, it may be possible to apply an adaptive grid strategy to obtain grid independent results without excessive computational cost.

\section{DISCUSSION}

For rectangular injectors, the penetration depth and measured mixing increase with aspect ratio (L/W). Again, this is largely due to a sustained generation of streamwise vorticity over a relatively long streamwise length. This improved vorticity production is likely attributed to a buffering effect, in which the aft portion of the injected flow is shielded from the mainstream by the streamwise vortices, which originate along the forward portion of the injector. The measured discharge coefficient and induced wall force also increase with aspect ratio, though the increase in measured wall force may simply be a function of increased discharge.

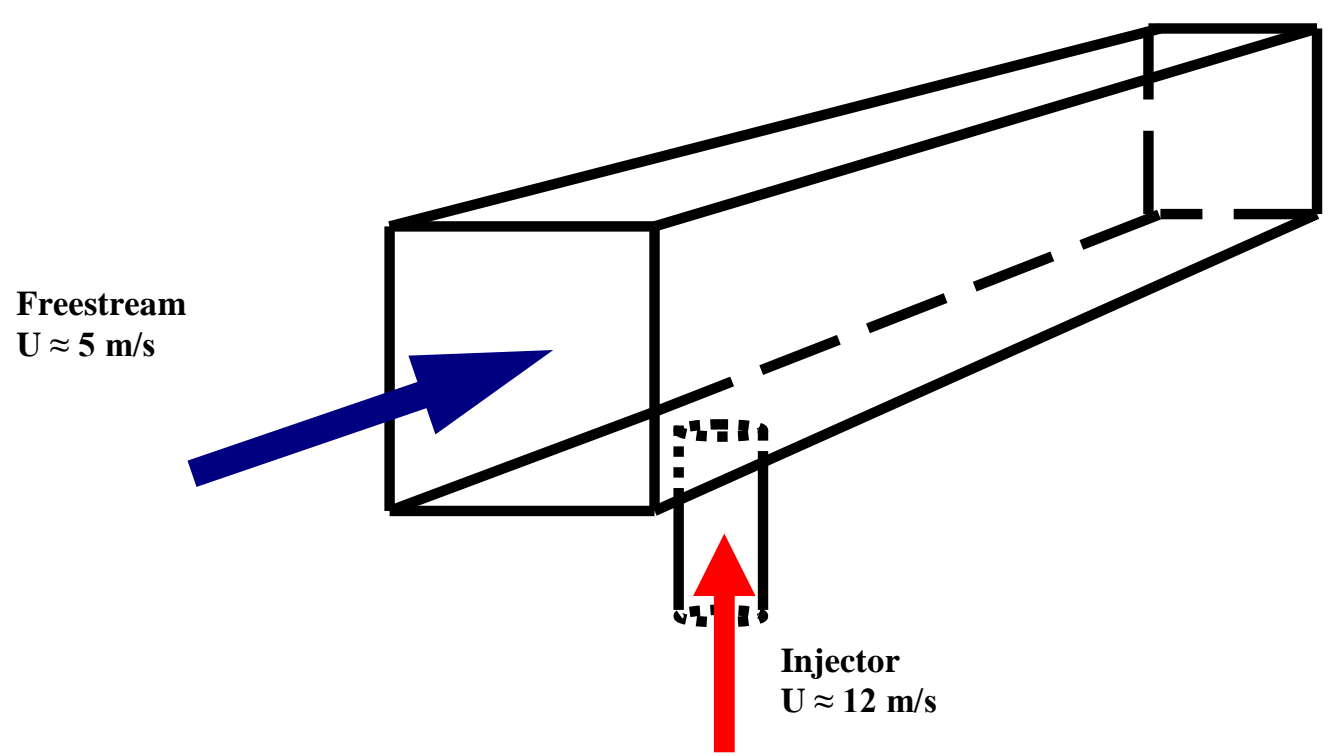

Fig 1: Subsonic Duct with Transverse Injector 


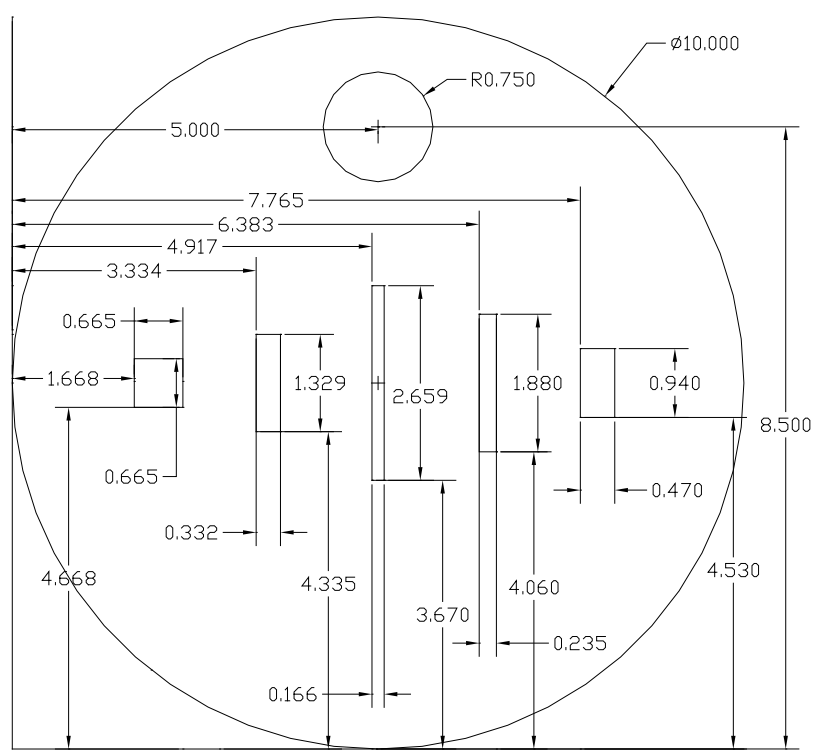

Fig 2: Injector Orifice Geometries/Injector Plate

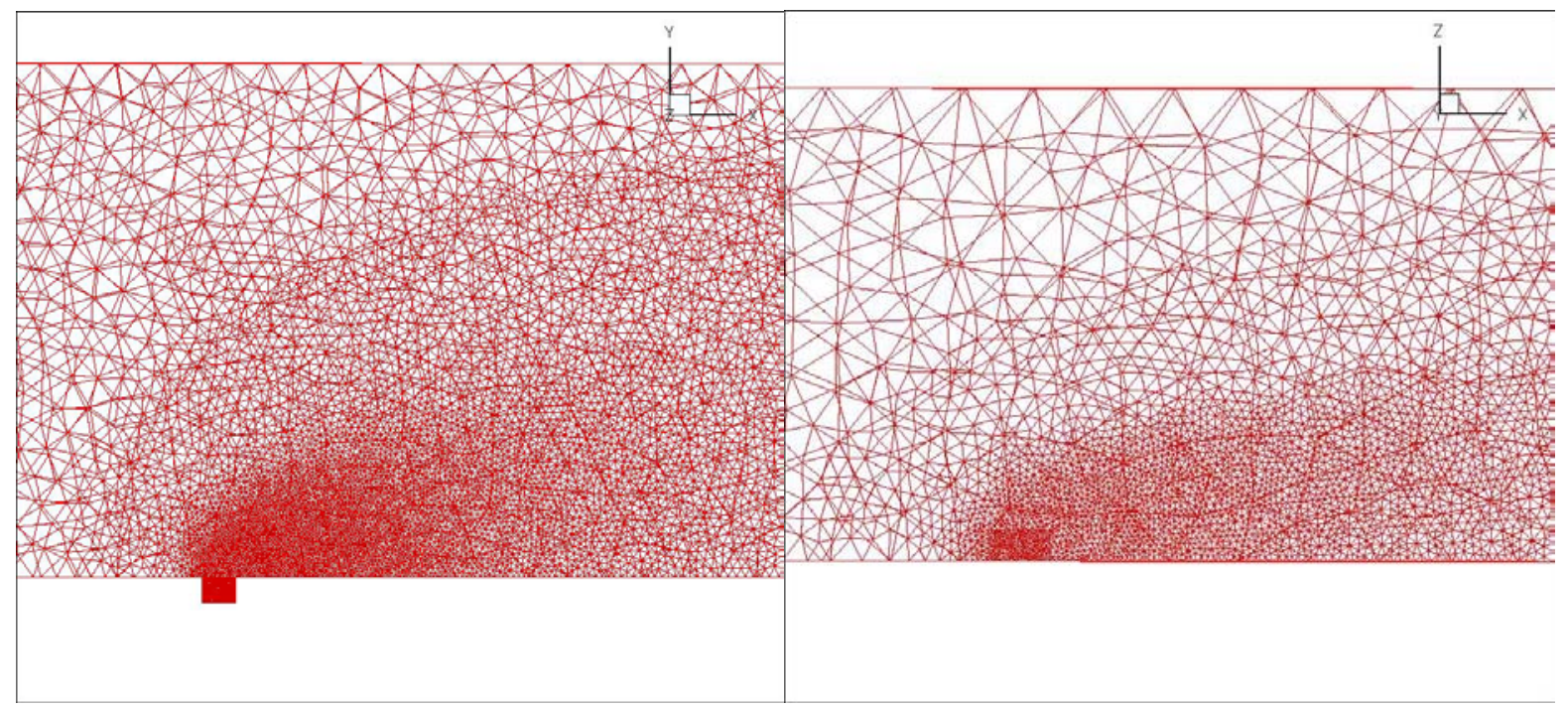

American Institute of Aeronautics and Astronautics 


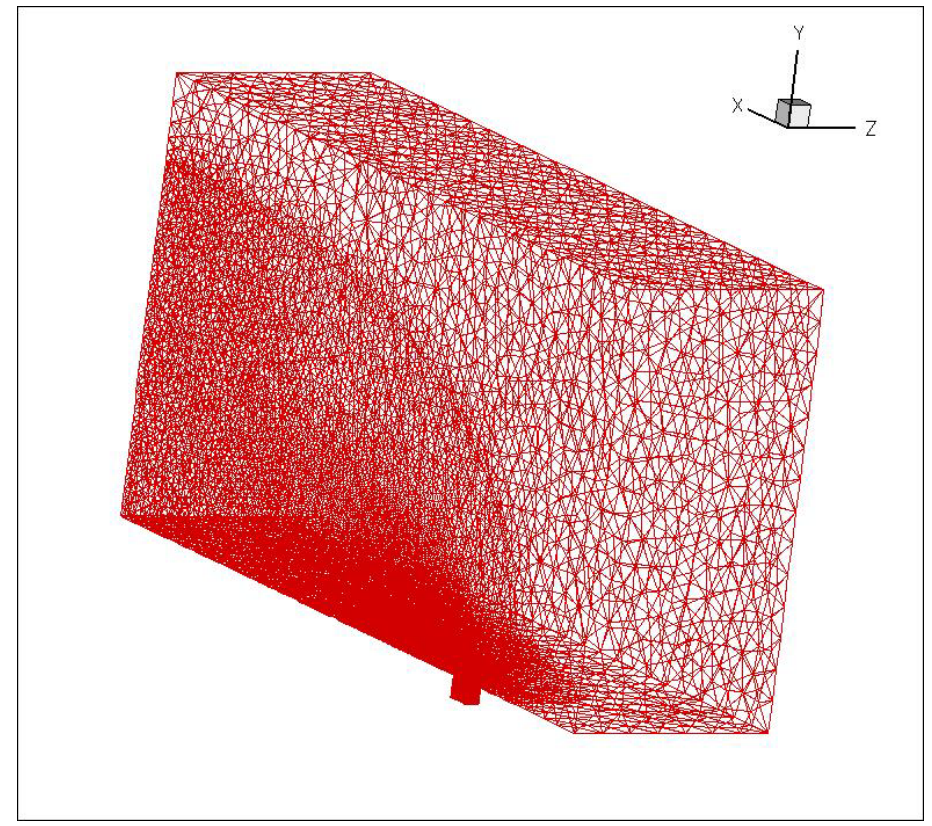

Fig 3: Injector Grid

5

American Institute of Aeronautics and Astronautics 


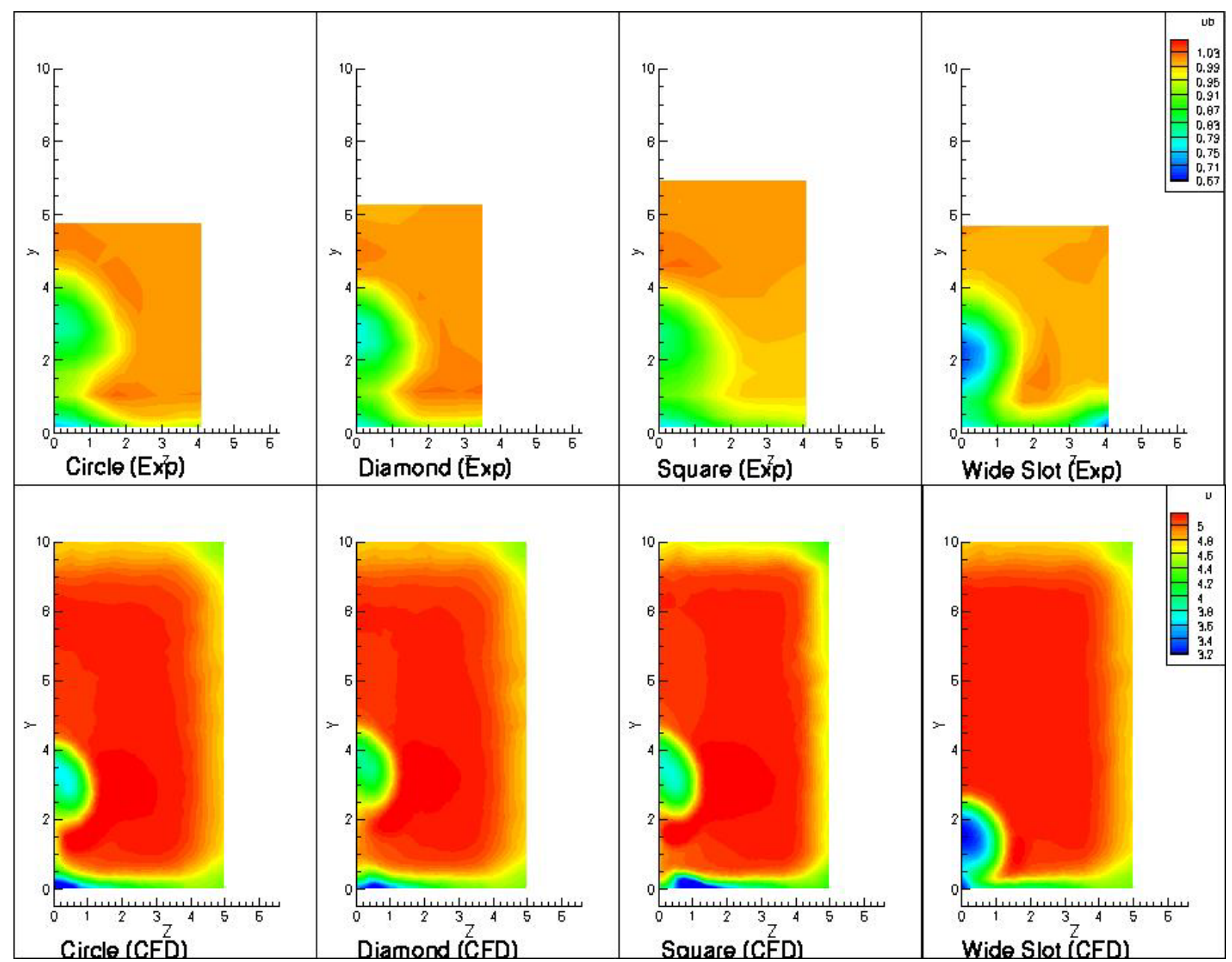

Fig 4: Axial Velocity at $x / D=10$

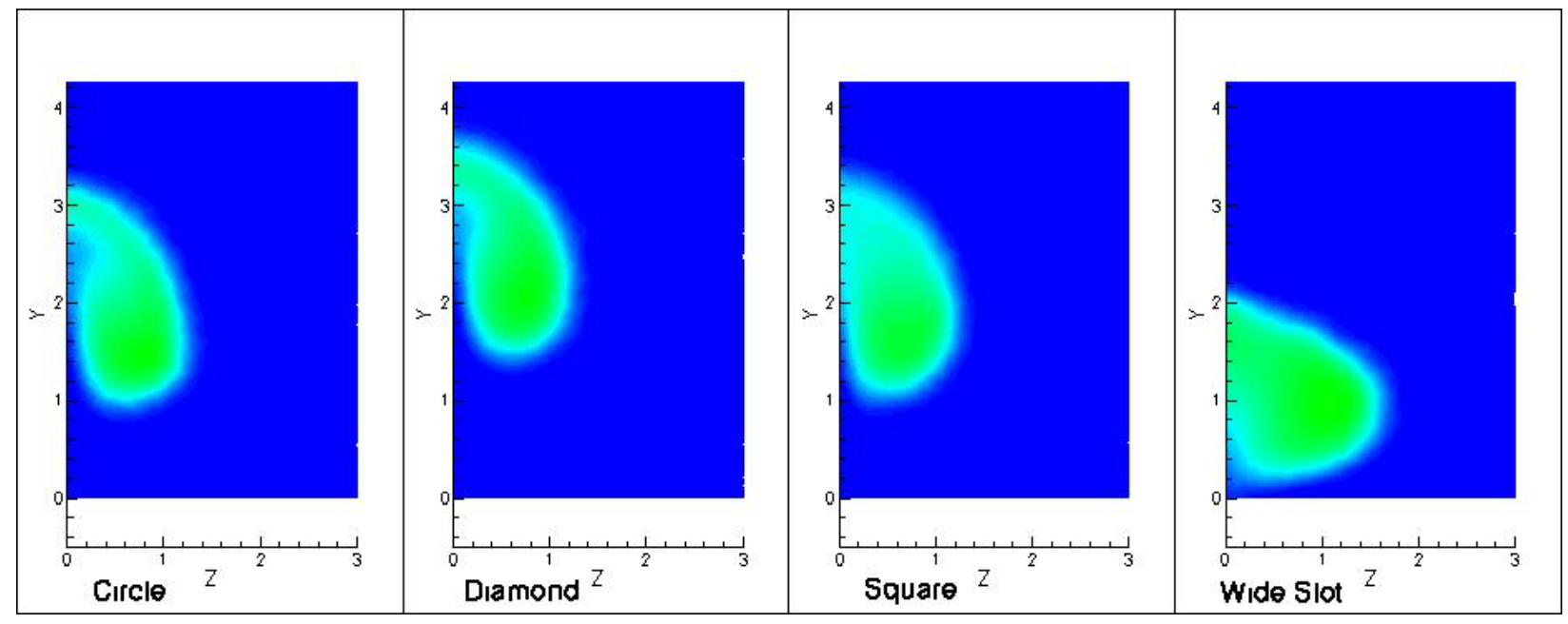

Fig 5: Injectant Mass Fraction at $\mathrm{x} / \mathrm{D}=5$ (CFD)

6

American Institute of Aeronautics and Astronautics 


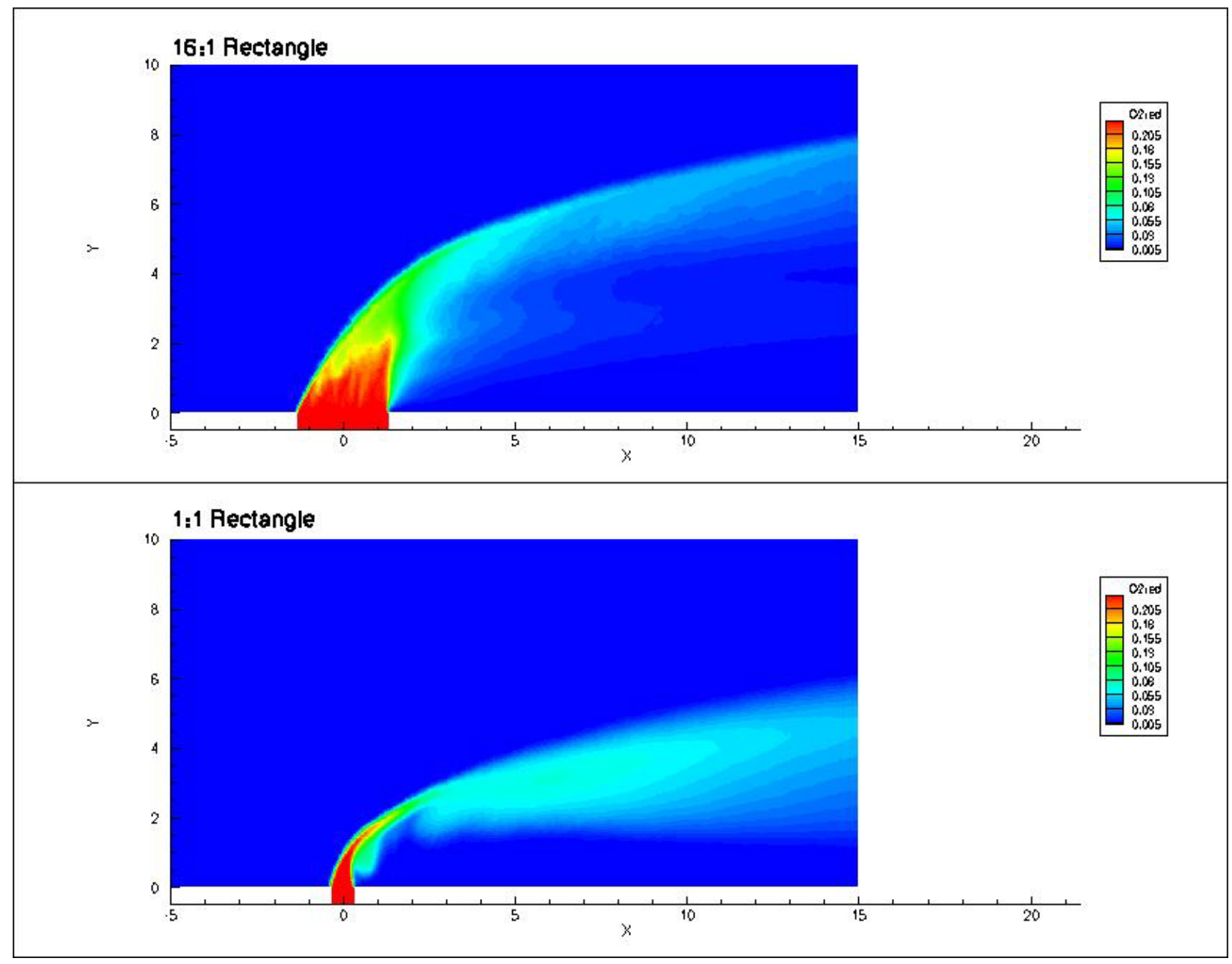

Fig 6: Injectant Mass Fraction at $\mathrm{z}=0$ (center of duct) 


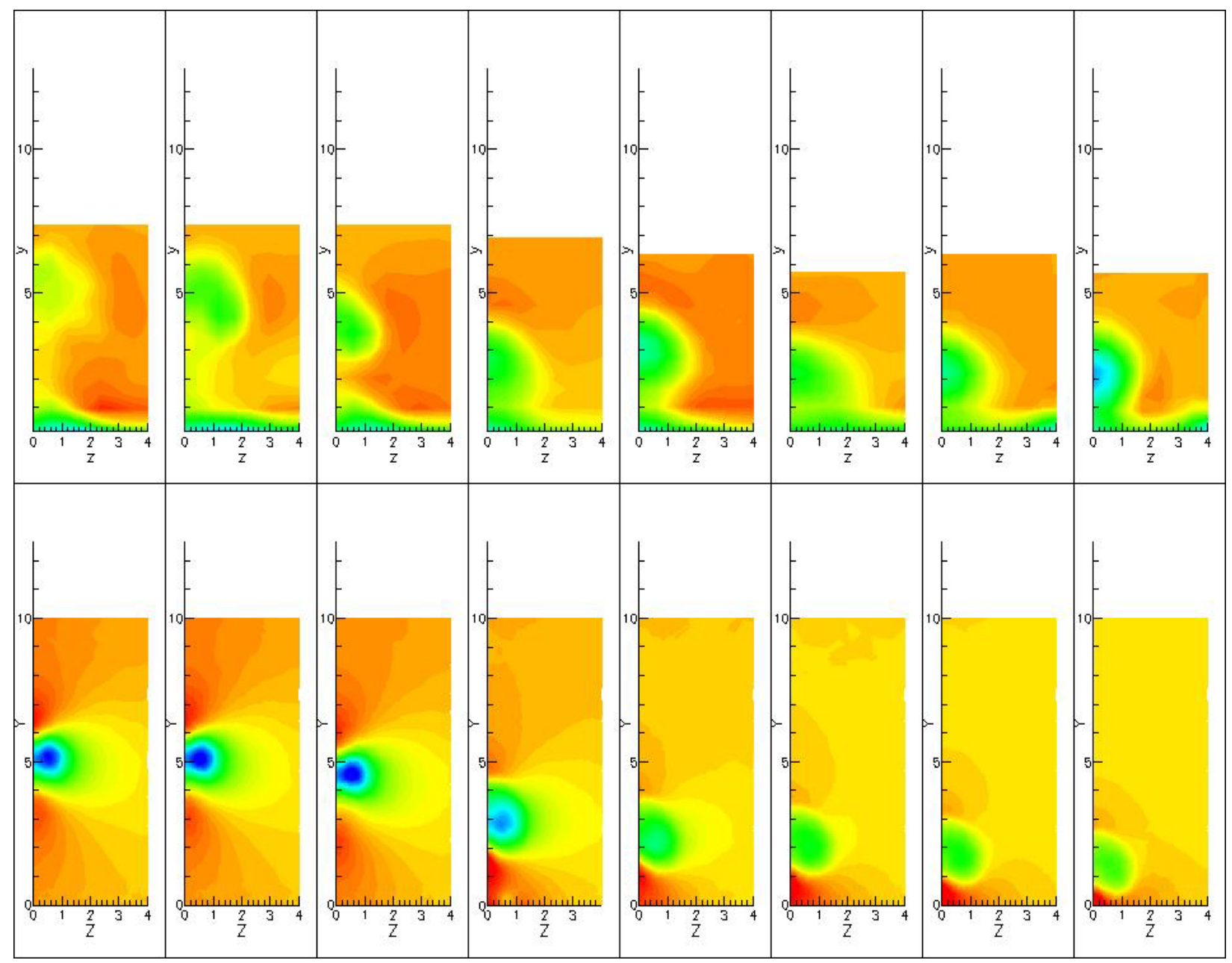

Fig 7: Axial Velocity of Rectangular Injectors with descending $L / W$ ratio at $x / D=10$ (Exp and CFD)

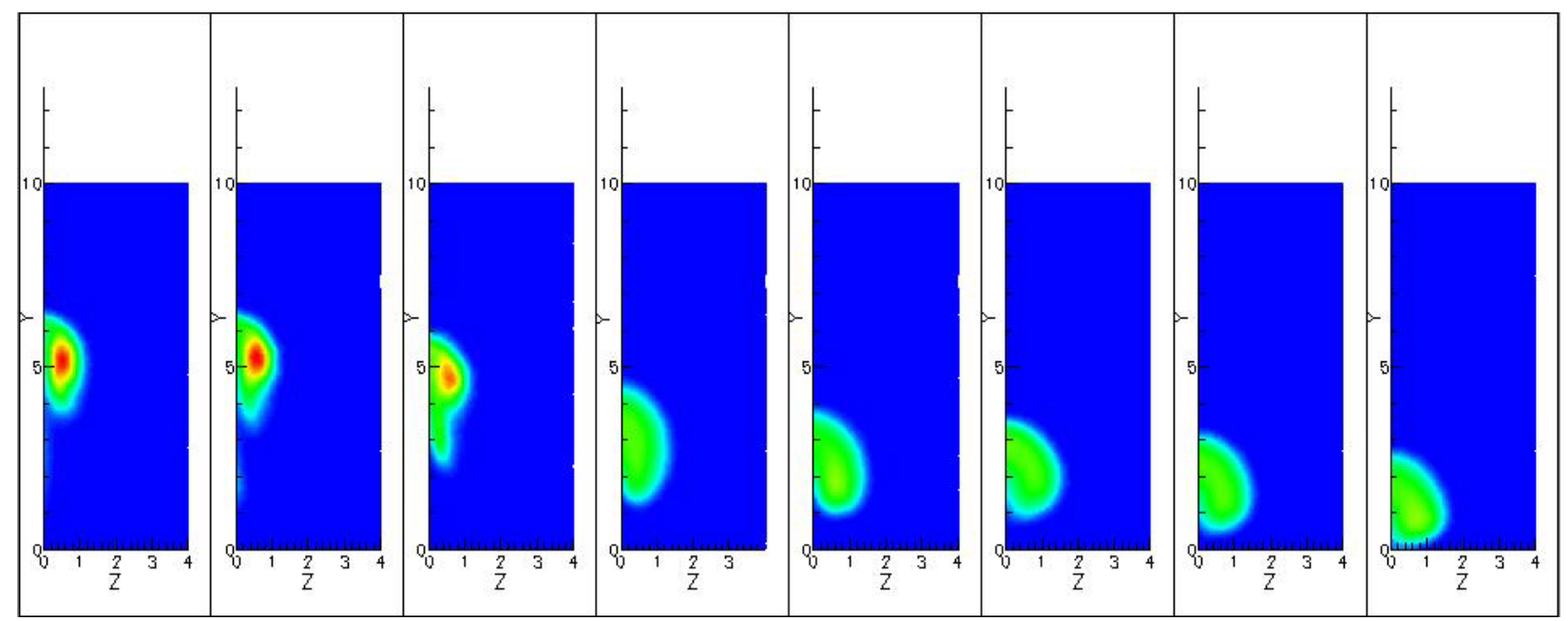

Fig 8: Injectant Mass Fraction of Rectangular Injectors with Descending $L / W$ ratio at $x / D=10$ (CFD) 
American Institute of Aeronautics and Astronautics 